\title{
Article \\ Layer-by-layer functionalized nanotube arrays: A versatile microfluidic platform for biodetection
}

\author{
Allison L. Yost ${ }^{1}$, Setareh Shahsavari ${ }^{1}$, Grinia M. Bradwell ${ }^{2}$, Roberta Polak ${ }^{3}$, Fabio Fachin ${ }^{4}$, Robert E. Cohen ${ }^{3}$, Gareth H. McKinley ${ }^{1}$, \\ Mehmet Toner ${ }^{5}$, Michael F. Rubner ${ }^{2}$ and Brian L. Wardle ${ }^{4}$
}

We demonstrate the layer-by-layer (LbL) assembly of polyelectrolyte multilayers (PEM) on three-dimensional nanofiber scaffolds. High porosity (99\%) aligned carbon nanotube (CNT) arrays are photolithographically patterned into elements that act as textured scaffolds for the creation of functionally coated (nano)porous materials. Nanometer-scale bilayers of poly(allylamine hydrochloride)/poly(styrene sulfonate) (PAH/SPS) are formed conformally on the individual nanotubes by repeated deposition from aqueous solution in microfluidic channels. Computational and experimental results show that the LbL deposition is dominated by the diffusive transport of the polymeric constituents, and we use this understanding to demonstrate spatial tailoring on the patterned nanoporous elements. A proof-of-principle application, microfluidic bioparticle capture using N-hydroxysuccinimide-biotin binding for the isolation of prostate-specific antigen (PSA), is demonstrated.

Keywords: layer-by-layer; carbon nanotube; microfluidic; nanoporous; functionalization; bioparticle capture

Microsystems \& Nanoengineering (2015) 1, 15037; doi:10.1038/micronano.2015.37; Published online: 21 December 2015

\section{INTRODUCTION}

The ability to achieve nanoscale control of interfaces and surfaces has fueled new ideas and technologies in a myriad of applications, including the design of various microelectronic ${ }^{1-3}$, energy storage $^{4-7}$, optical ${ }^{8-11}$, and biomedical devices ${ }^{12-15}$. In particular, biomedical research has benefited from advances in nanoscale surface chemistry and surface manipulation, enabling applications including the controllable adsorption/release of proteins, affinity chromatography, and drug delivery ${ }^{16-18}$. The vast majority of surface tailoring, however, has been demonstrated on readily accessible planar or particle surfaces, and the transition to more complex three-dimensional (3D) porous materials, particularly space-constrained nanoporous materials, is currently limited. An essential advance to enable the production of the next generation of biomedical devices is the ability to systematically functionalize the surfaces of 3D nanotemplates contained within complex geometries with conformal nanoscale coatings of any desired chemistry and surface morphology. The conformality of the resultant coating and its precise nanoscale thickness is critical to maintaining the advantages and control of functionality provided by the original 3D nanoscale template.

A promising nanostructuring approach to create $3 \mathrm{D}$ templates is the use of textured nanoporous elements as scaffolds for surface functionalization and as building blocks for various devices. One such textured element that has received considerable attention is an aligned array of nanofibers, such as carbon nanotubes (CNTs) ${ }^{19-21}$. CNTs are attractive because of their intrinsic mechanical, electrical, magnetic, and optical properties ${ }^{22-29}$ as well as the multiple routes to synthesize and give texture to bulk assemblies of the nanofibers. Biomedical applications are particularly well suited, owing to the wealth of existing surface chemistries available for functionalization (e.g., antibody binding). As such, one can apply nanoscale control over chemical composition, spatial morphology, and the interfaces of materials to create versatile, materials-driven platform technologies that can be targeted to a wide range of applications. For example, by exploiting the high porosity (99\%) of CNT arrays, vertically aligned CNT (VACNT) forests can be successfully integrated into a variety of devices ${ }^{30,31}$, including within microfluidic devices, allowing the separation and specific targeting of biomolecules ranging in size from $40 \mathrm{~nm}$ to $10 \mu \mathrm{m}$ with an enhanced $(\times 7)$ capture efficiency $^{32,33}$. Owing to their size and high surface area, CNTs provide unique accessibility to bioparticles in a high throughput fashion at scales currently difficult to achieve through microelectromechanical systems at such rates, such as biomarker proteins, viruses, exosomes, or cell-free DNA in the blood or other bodily fluids for diagnostic applications.

Although in principle, various approaches exist for modifying the surfaces of CNTs contained within CNT arrays, the layer-bylayer (LbL) assembly approach ${ }^{34}$ holds the most promise for fulfilling the multiple requirements of nanoscale thickness, morphology control, conformal coating uniformity, and the ability to create a wide range of different surface chemistries and functionalities. Indeed, the greatest advantage of the LbL assembly process compared to other coating processes continues to be its ability to produce nanoscale conformal coatings from an extremely wide variety of organic, inorganic, and biological molecules and materials ${ }^{35-38}$. Excellent examples of these capabilities as applied to biomaterial-based devices and constructs including sensors ${ }^{39}$ and drug delivery elements ${ }^{40,41}$ abound in the literature. Relevant to this work is the idea that LbL assembly can be readily carried out within the confines of complex nanoscale geometries and within functional microfluidic

\footnotetext{
${ }^{1}$ Department of Mechanical Engineering, Massachusetts Institute of Technology, 77 Massachusetts Avenue, Cambridge, MA 02139, USA; ${ }^{2}$ Department of Materials Science and Engineering, Massachusetts Institute of Technology, 77 Massachusetts Avenue, Cambridge, MA 02139, USA; ${ }^{3}$ Department of Chemical Engineering, Massachusetts Institute of Technology, 77 Massachusetts Avenue, Cambridge, MA 02139, USA; ${ }^{4}$ Department of Aeronautics and Astronautics, Massachusetts Institute of Technology, 77 Massachusetts Avenue, Cambridge, MA 02139, USA and ${ }^{5}$ Center for Engineering in Medicine and BioMEMS Resource Center, Massachusetts General Hospital, Harvard Medical School, and Shriners Hospital for Children, Boston, MA 02139, USA
}

Correspondence: Brian L. Wardle (wardle@mit.edu)

Received: 10 September 2015; Revised: 2 November 2015; Accepted: 2 November 2015 
devices ${ }^{42}$. In the former case, LbL assembly has been demonstrated within nanochannels and nanopores ${ }^{42-46}$. In the latter case, LbL-assembled coatings have been used for the development of microfluidic-based in vitro assays or for studying fundamental cell and tissue biology. For example, Sung et al. have modified polydimethylsiloxane surfaces by using LbL coatings to reduce non-specific binding and enhance the detection of low levels of protein ${ }^{47}$. Others have designed a microfluidic platform with LbL coatings for identifying the dengue virus through an enzyme-linked immunosorbent assay (ELISA) approach. The results have indicated that $\mathrm{LbL}$ coatings on channel surfaces improve their stability and efficiency, reducing surface modification time 12 -fold ${ }^{48}$. Alternatively, LbL-modified microfluidic systems have been used to control the flow or flow constituents within the device. Kirchof et al., for example, have used LbL coatings in microfluidic devices to generate $\mathrm{pH}$ gradients that promote cell migration ${ }^{49}$, whereas Barker et al. have used them to alter surface charge and control the direction of electroosmotic flow ${ }^{50}$.

Our approach has been to utilize LbL coatings as a means to systematically functionalize patterned CNT arrays contained within microfluidic devices designed to filter, capture, and detect low levels of biological markers of disease $\mathrm{e}^{32,33}$. We demonstrate that it is possible to control the LbL assembly pattern within a microfluidic device by only varying the height of the microchannel. In this manner, we can coat and functionalize individual CNTs throughout the entire CNT array or cover just the outer surface of the array. In the former case, conformal coatings on individual nanotubes have been achieved within arrays with tube-to-tube spacings of approximately $80 \mathrm{~nm}$. This level of functionalization control can be used to take full advantage of the highly porous nature of the CNT arrays, as well their ability to controllably capture and release biomarkers of various types. To demonstrate this, we used this technology to functionalize the CNT surfaces with antibodies and capture prostate-specific antigen (PSA) as an exemplary capture target. A significant challenge in microfluidic platform bioparticle isolation is to achieve sufficient interaction between the target biomolecule and the functional surfaces to promote binding. This work provides a powerful tool to functionally tailor and grade nanomaterials in three dimensions, enabling the design of devices that enhance the bioparticle-surface interaction. Additionally, owing to the nature of the spacing between the individual CNTs ( $80 \mathrm{~nm})$, this platform specifically has high potential in the isolation of nano-sized bioparticles, such as viruses, exosomes, or DNA.

\section{MATERIALS AND METHODS}

The microfluidic chip design and assembly processes (Figure 1) were developed to favor adsorption and LbL conformal coating on the nanoporous CNT elements contained within the microfluidic device (Figure 1a). Patterned CNT elements were grown via chemical vapor deposition on Si wafers. (Fabrication details can be found in the Supplementary Information). These porous VACNT forests consist of arrays of multi-walled CNTs (OD: $7.78 \pm 0.85 \mathrm{~nm}$; ID: $5.12 \mathrm{~nm} \pm 0.76 \mathrm{~nm}$, spaced $\sim 80 \mathrm{~nm}$ apart, \# walls $5 \pm 1,1$ vol\%, $1.59 \mathrm{~g} \mathrm{~cm}^{-3} \frac{51,52}{}$ ) (Figure $1 \mathrm{~b}$ and $\mathrm{c}$ ) patterned by using photolithography into macroscopic elements that have high porosity ( 99\%) and accessibility to aqueous solutions. For this study, one geometric pattern of CNTs was used for simplicity: a single cylindrical pillar of $1 \mathrm{~mm}$ in diameter (consisting of more than $10^{8}$ individual carbon nanutubes) centered in the $3 \mathrm{~mm}$ wide, $7 \mathrm{~mm}$ long microfluidic channel (Figure $1 \mathrm{~b}$ and $\mathrm{c}$ ). CNT pillar heights averaged $50 \mu \mathrm{m}$ but
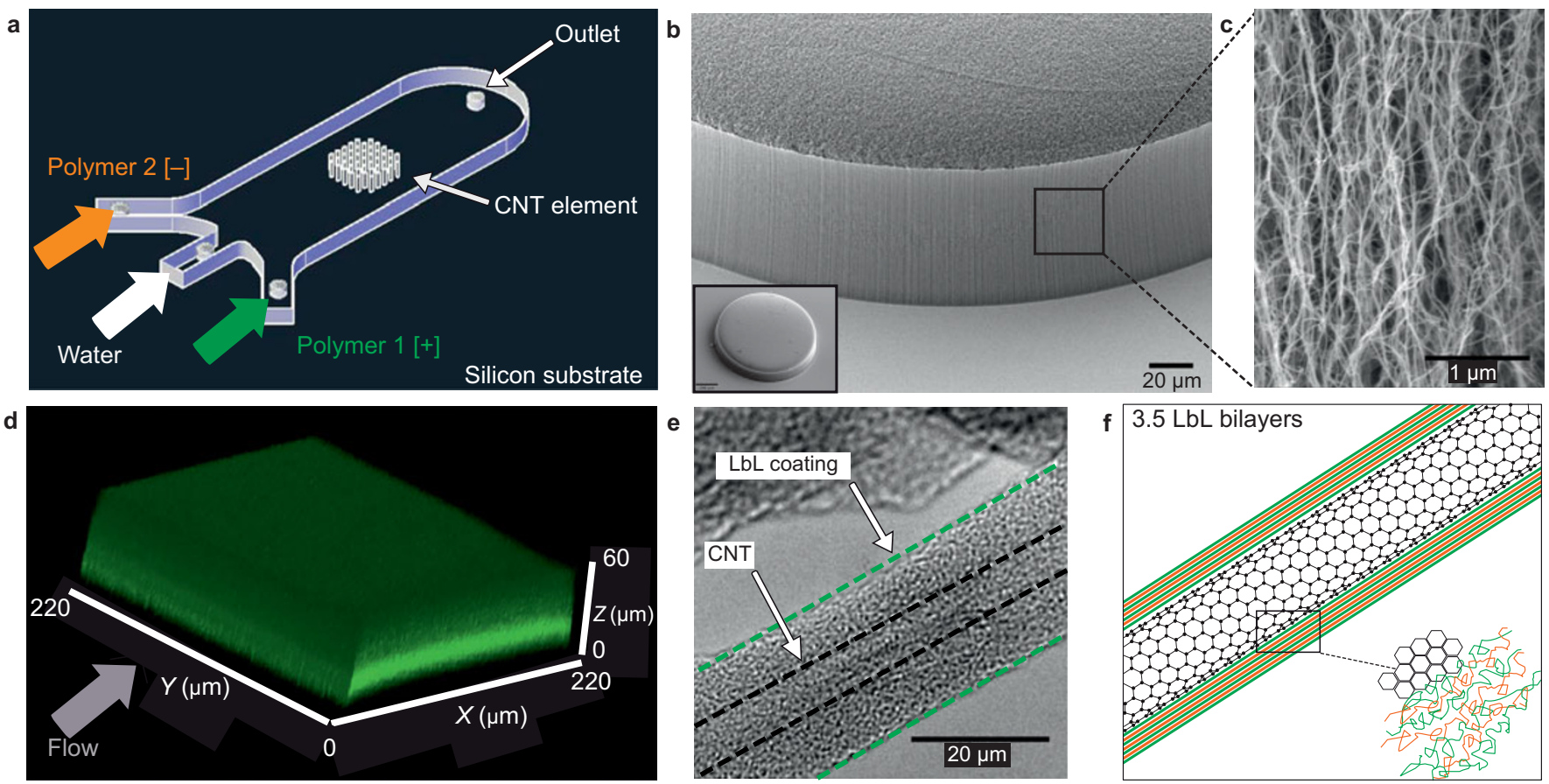

Figure 1 Schematic and characterization of LbL coating on a cylindrical-based nanoporous CNT element. (a) Illustration of microfluidic chip design featuring a pillar $(D=1 \mathrm{~mm})$ CNT element in the microfluidic channel ( $3 \mathrm{~mm}$ wide). (b) Scanning electron microscope (SEM) images of an as-grown aligned CNT pillar element. (c) High-resolution SEM of CNT element exhibiting textured porosity. (d) 3D reconstruction of confocal Z-slices (of poly(allylamine) hydrochloride (PAH)-Fluor, $488 \mathrm{~nm}$ ) of a $220 \times 220 \mu \mathrm{m}$ section of the cylindrical CNT pillar element, demonstrating the polymer coating through each vertical section. (e) Transmission electron microscopy (TEM) micrograph of an individual CNT coated with 3.5 bilayers of PAH-Fluor/SPS assembled at pH 9.3. The dashed black line outlines the CNT diameter, and the green dashed line indicates outer edge of the LbL coating. (f) Illustration of 3.5 LbL bilayers. 
ranged between $30 \mu \mathrm{m}$ and $80 \mu \mathrm{m}$. Results were also obtained with other element geometries (not shown), such as an array of pillars or a porous "wall", i.e., a porous rectangular feature arranged perpendicular to flow. In fact, for particle isolation, a partitioned microfluidic device containing a nanoporous wall, which acts like a filter, would be an ideal design; however, the resulting pressure drop across the dividing wall can damage the adhesion of the CNTs to the substrate. Therefore, our cylindrical geometric design resolves the trade-off between the maximum allowed pressure and maximum interception of flow streamlines with the CNT elements. Photolithography permits numerous patterns to be designed in the future for further investigation of the optimal nanoporous CNT element geometries.

Microfluidic channels were fabricated according to standard soft lithography protocols $s^{53}$ in conjunction with negative molds made of SU-8 photoresist (Microchem, MA, USA) onto silicon wafers. The microfluidic chip design had three inlets: one for each polyelectrolyte solution used in the LbL process and one for a water rinse required to wash out the polymer molecules that are not adsorbed in each step onto the CNTs (Figure 1a).

Polyelectrolyte multilayer (PEM) film deposition on the CNT surfaces was performed via the LbL assembly of PAH (15 kDa) and poly(styrene sulfonate) (SPS; $70 \mathrm{kDa}$ ) within the microfluidic devices. PAH conjugated with fluorescein (PAH-Fluor) was used to permit visualization. Integrated CNT microfluidic devices were primed with a negatively charged surfactant (sodium dodecyl benzene sulfonate, SDBS), which was selected for its propensity to bind to individual CNTs ${ }^{54}$ and its electrical charge that favors polyelectrolyte adsorption. Because CNTs have a tendency to be intrinsically hydrophobic, the SDBS served to lower the surface energy of the nanotubes, making the CNT elements more hydrophilic and more receptive to polymer coating ${ }^{55,56}$. After priming, the devices were rinsed with DI water for $5 \mathrm{~min}$ at $6 \mu \mathrm{L} \min ^{-1}$, and LbL assembly was performed via the alternate flow of PAH-Fluor and SPS solutions at $6 \mu \mathrm{L} \mathrm{min}{ }^{-1}$ for 5 min each, with intermediate water rinse flow $\left(5 \mathrm{~min}, 6 \mu \mathrm{L} \mathrm{min}{ }^{-1}\right)$. PAH-Fluor was the first and last layer deposited to retain a positive charge and because it was labeled for imaging.

As previously described, PAH/SPS films assembled at $\mathrm{pH} 9.3$ present primary amine groups isolated in hydrophobic pockets that can be opened via an acid treatment that will protonate the amine groups and expose them to the surface of the film ${ }^{57,58}$. The exposed amine groups can be reacted with selected chemistries, such as N-hydroxysuccinimide (NHS) groups, creating stable amide bonds to link a biotinylated surface to the film surface ${ }^{59}$. Thus, $\mathrm{pH} 9.3$ was selected for the standard LbL assembly, and an acid treatment at $\mathrm{pH} 2.5$ was performed after the LbL deposition and prior to the bioparticle capture protocol to generate aminerich surfaces. LbL assembly was performed successfully with 3.5 bilayers of PAH-Fluor/SPS at $\mathrm{pH} 9.3$ via continuous flow LbL assembly.

\section{RESULTS AND DISCUSSION}

We first sought to achieve full, uniform coatings throughout the microfluidic $1 \mathrm{~mm}$ pillar CNT device. The full CNT pillar element coating was achieved when devices were fabricated and assembled such that there was a gap $(\sim 50 \mu \mathrm{m})$ between the CNT element height and the PDMS channel height. Confocal microscopy images showed a polymer coating throughout the entire volume of the CNT element (Figure 1d), and TEM micrographs suggested nanoscale conformal coating layers on individual CNTs within the element by comparing the CNT diameters before and after the LbL deposition (Figure 1e), confirming the ability to coat the individual CNTs and construct a 3D LbL-coated surface. It is notable that there tended to consistently be a higher intensity of fluorescence signal in the mid Z-plane of the device, as shown in Figure $1 \mathrm{~d}$, suggesting a larger amount of coating in that location. We believe this is an artifact of the rinsing methods used, which removes some polymer from the top surface of the device. Additionally, it is possible that this intensity variation is diagnostic of the CNT morphology, and perhaps the changes in the CNT density along the $Z$-axis give rise to small changes in polymer coating. It is also possible that this variation is due to an imaging artifact based on the use of a laser scanning confocal microscope to image through the PDMS channel, which causes scattering and difficulty in focusing far away from each boundary.

Because a uniform, conformal coating was achieved with a gap in the device geometry, we hypothesized that the presence of this gap was important in controlling the coating morphology. We designed devices such that there was no clearance gap between the CNTs and the top of the PDMS channel by slightly compressing the CNT element along the $Z$-axis (reference axis in Figure $1 \mathrm{~d}$ ). The results in this modified geometry showed only an annular coating of the CNTs. The thickness of the annular coating was on the order of half the height of the CNT element. This annular coating mechanism was maintained throughout the volume of the CNT element and was distinctly different from the full coating results obtained when a clearance gap was maintained (Figure 2). Thus, by changing the geometry of the device elements, we were able to exhibit spatial control of the LbL coating. Furthermore, methods have been investigated to close a PDMS gap in situ ${ }^{60}$ such that the channel could be sealed after the LbL coating but prior to any biological or chemical assay.

To describe the difference in the uniform vs. annular coating mechanisms, a 3D numerical model was developed to investigate the transport of the polymer solution through the CNT elements. The transport phenomena involved in the experiments included convection, diffusion, and adsorption. In our numerical model, we included convection and diffusion but neglected adsorption because the timescale for the adsorption is much smaller than that of diffusion ${ }^{61}$. Experimental observations suggested that as soon as the polymer solution diffuses into the CNT forest, the polymer is adsorbed onto the CNT surface. To further quantify the kinetics of the adsorption, we calculated the Damköhler number, Dk, which compares the reaction rate with the rate of diffusive transport ${ }^{62}$ :

$$
\mathrm{Dk}=\frac{\text { time scale of diffusion }}{\text { time scale of reaction }}
$$

Here, the reaction of interest is the adsorption of the long-chained polymer molecules with charged groups to the surface with opposite polarity. On the basis of the theoretical analysis by Stuart et al. ${ }^{61}$ equilibrium in adsorption should be attained on a timescale of seconds in our system. In our experiments, the diffusion occurred on a timescale of minutes to hours; therefore, the Damköhler number was in the range of 100-1000, which justifies neglecting the adsorption kinetics in the numerical model.

For a quantitative comparison of the rate of the polymer transport by convection and diffusion, we determined the Péclet number, defined as:

$$
\mathrm{Pe}=U_{c} L_{c} / D_{12}
$$

where $U_{c}$ and $L_{c}$ are the characteristic velocity and length scale, respectively, and $D_{12}$ denotes the binary diffusion coefficient of species 1 (polymer) and solvent 2 (water). In our system, two Péclet numbers existed owing to the two different velocities as a result of the different structural length scales: one based on the height of the microchannel and the other based on the pore size in the CNT forest. Inside the nanoporous domain, the characteristic velocity can be obtained by comparing the hydrodynamic 

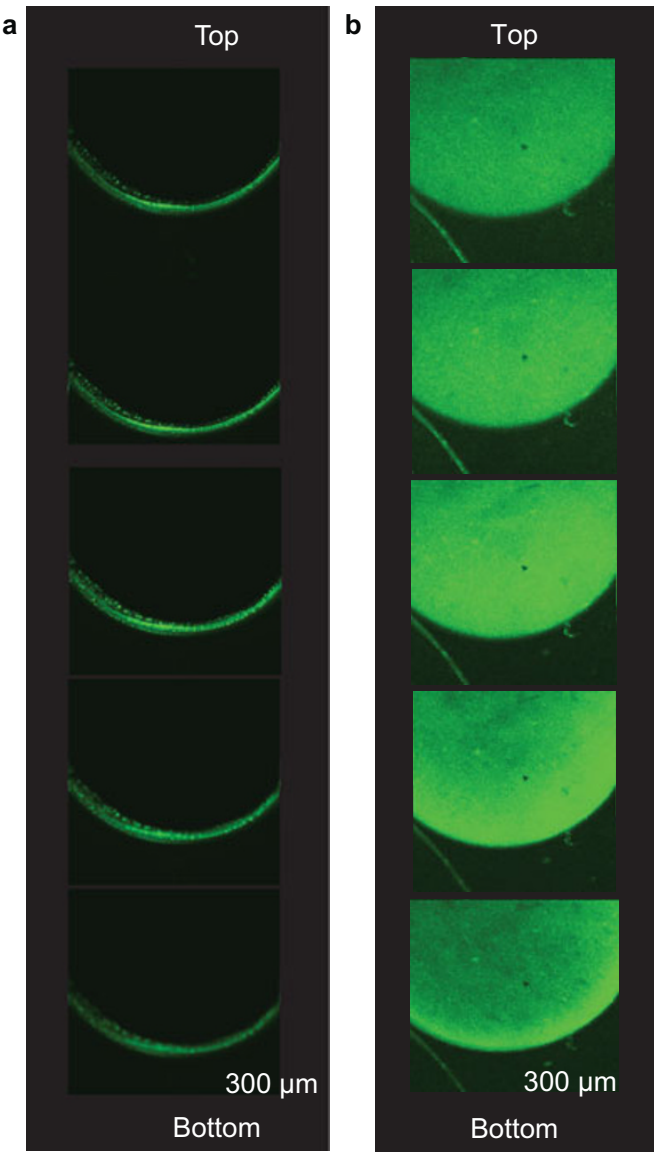

C

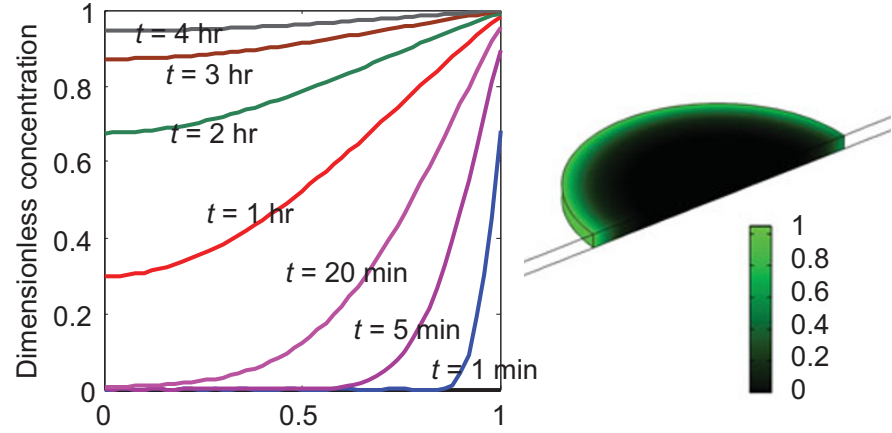

Dimensionless radial coordinate

d

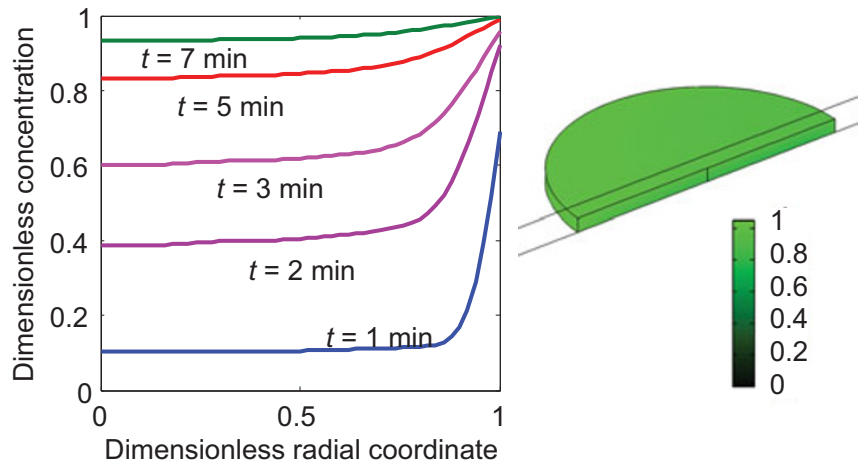

Figure 2 Spatial control of LbL multilayer deposition. (a) Confocal Z-slices in $10 \mu \mathrm{m}$ increments of a section of the cylindrical CNT device with no clearance gap in microchannel (PEM assembly performed with (PAH9.3/SPS9.3) 3.5 ) after a 5 min flow of the polymer solution. (b) Confocal Z-slices in $10 \mu \mathrm{m}$ increments of a section of a CNT device with a $50 \mu \mathrm{m}$ clearance gap in microchannel (PEM assembly performed with (PAH9.3/SPS9.3) 3.5 $_{\text {) }}$ after a 5 min flow of the polymer solution. (c) 3D numerical simulation of polymer concentration for a device with no gap. (d) 3D numerical simulation of polymer concentration for an identical device with a $50 \mu \mathrm{m}$ clearance gap above the array of pillars.

resistances of the porous CNT pillar element and the side gap between the pillar and the channel wall. The order of magnitude for the average velocity through the side gap can be estimated as $U \sim \frac{H^{2}}{\mu} \frac{\Delta p}{L}$, where $H$ is the channel height, $\mu$ is the fluid viscosity, and $\Delta p / L$ is the pressure drop over the length of the channel, $L$. Additionally, according to Darcy's law, the apparent velocity inside the porous CNT forest is $U_{p}=\frac{k}{\mu} \frac{\Delta p}{L}$, where $k$ is the permeability of the CNT forest. Therefore, the ratio of the two velocities is $\frac{U_{p}}{U}=\frac{k}{H^{2}}=10^{-8}$ (using $H=100 \mu \mathrm{m}$ and $k \sim 10^{-16} \mathrm{~m}^{2}$, derived from Ref. 63). In our experiments, the channel flow velocity is $U=3 \times 10^{-4} \mathrm{~m} \mathrm{~s}^{-1}$ (corresponding to a flow rate of

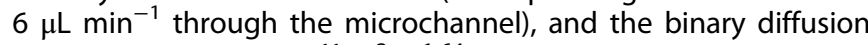
coefficient is $D_{12}=10^{-11} \mathrm{~m}^{2} \mathrm{~s}^{-164}$. Therefore, the Péclet number in the channel is $\mathrm{Pe}=\frac{U H}{D_{12}}=3 \times 10^{3}$, whereas in the CNT forest, the relevant dimensionless ratio is $\mathrm{Pe}_{p}=\frac{U_{p} H}{D_{12}}=3 \times 10^{-5}$. The high disparity between these values clearly indicates that outside the porous CNT pillar, convection is dominant whereas inside the CNTs, diffusion controls the transport of polymer molecules in spite of the high porosity (99\%) of the CNTs.

In fact, the flow permeation inside the pillar is negligible as a result of two contributing factors. The first is the low Darcy number of the nanoporous medium, Da. Da is defined as the permeability, non-dimensionalized by the porous collector diameter, $(D)$ :

$$
\mathrm{Da}=\frac{k}{D^{2}}
$$

which yields a value of $\mathrm{Da}=10^{-10}$ for our system. The second is the low confinement ratio, the ratio of the collector diameter $(1 \mathrm{~mm})$ to the channel width $(3 \mathrm{~mm})$. The flow permeation through confined cylindrical porous collectors has been studied by Shahsavari et al. According to Shahsavari and McKinley ${ }^{65}$, the superficial velocity in the porous region relative to the free stream velocity should be on the order of $10^{-8}$ for our geometrical parameters, which is the same result that we estimated by comparing the hydrodynamic resistance of the channel and the porous CNT pillar. Ultimately, the combination of these two factors suggests that the porous CNT collector is essentially impermeable, owing to the dominance of convective effects when $\mathrm{Pe} \geq 1$. Further details regarding the numerical simulation can be found in the Supplementary Information.

The experimental findings (Figure $2 \mathrm{a}$ and $\mathrm{b}$ ) were consistent with the resulting concentration distributions based on the numerical modeling (Figure $2 \mathrm{c}$ and $\mathrm{d}$ ), which explain the two separate and distinct coating patterns observed experimentally. The numerical modeling confirmed that the assembly process is dominated by diffusion, predicting that for a microfluidic device with a controlled gap on top of the pillar, the polymer molecules diffuse axially through a thickness of only $\sim 50 \mu \mathrm{m}$, whereas in the device with no gap clearance, the polymer molecules can only diffuse radially. The experimental confocal microscopy results showed that this radial, annular coating penetrated radially to a distance on the order of $50 \mu \mathrm{m}$ as well. 
We used this understanding to functionalize the CNT surfaces with antibodies and capture PSA as an exemplary capture target. A modified sandwich ELISA on cylindrical CNT elements coated with 3.5 bilayers was performed to capture PSA $\left(100 \mathrm{ng} \mathrm{mL}^{-1}\right)$ by using a biotinylated secondary antibody and streptavidin-coated quantum dots (Qdots) (605 nm; Figure 3a). All capture and control experiments were performed on devices with a gap, thus using a full spatial LbL coating (further information on assay located in Supplementary Information). Confocal microscopy results indicated a high correlation between the LbL coating signal (fluorescein isothiocyanate (FITC), $488 \mathrm{~nm}$ ) and the capture signal (Qdots, $605 \mathrm{~nm}$ ), and capture was observed throughout the volume of the device (through Z-plane Figure $3 \mathrm{~b}$ ). Particles were advantageously captured throughout the element volume rather than solely on the outer surface, as in typical microfluidic capture elements. Intensity measurements were collected across multiple devices and Z-plane stacks with ImageJ, and the average ratio of signal to background noise was determined. The LbL functionalization approach yielded a capture rate 1.4 times higher than all the CNT element controls, which demonstrates that specific, covalent capture was achieved within the CNT elements.
The functionalization protocol $^{59}$ used herein has only recently been reported and has not yet been optimized for the CNT scaffolds. The capture protocol demonstrates the broad utility of the 3D LbL coating platform on CNT elements rather than a specific ability to target PSA ${ }^{66}$. Tailoring devices with sufficient physical interactions between bioparticle targets and the functionally coated surfaces is a great challenge in microfluidic bioparticle isolation. By using this platform, we increased the surface area by a factor of 20 for an equivalent microfluidic channel volume with a planar LbL coating, providing an enhanced sensing platform that is compatible with current microfluidic platforms.

Owing to the conformality of the LbL coating, the approach reported here can also be used to control the intra-CNT spacing within the 3D element, enabling both physical and surface chemistry tailoring. Porous CNT scaffolds allow isolation of particles over three orders of magnitude in size with a single chip $^{33}$ : particles that are larger than the average intra-CNT spacing $(\sim 80 \mathrm{~nm})$ do not penetrate into the CNT elements and can be isolated on the features' external surfaces via mechanical filtration and/or chemical affinity; particles that are smaller than
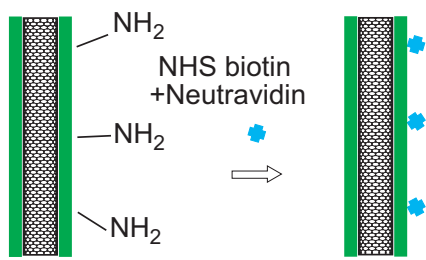

+Anti-PSA antibody biotinylated

$<$

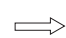

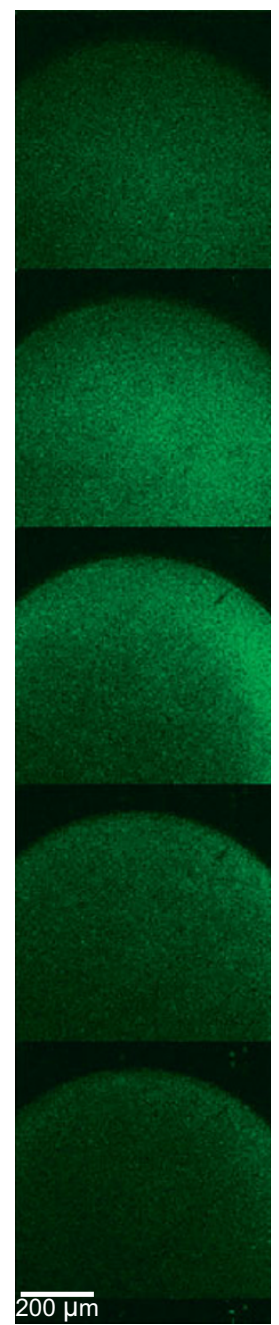

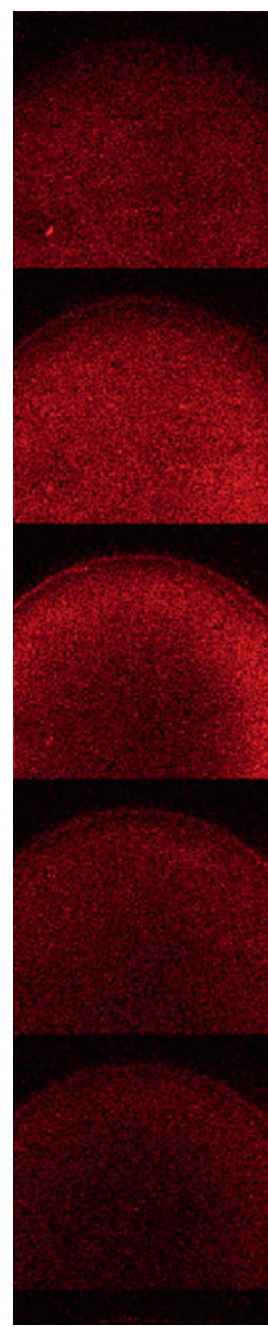

C

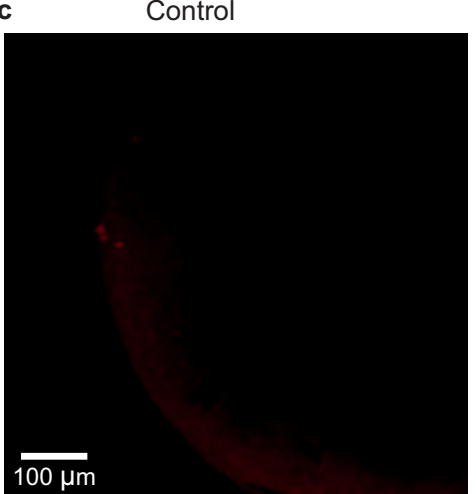
dots 655 streptavidin conjugated

凶
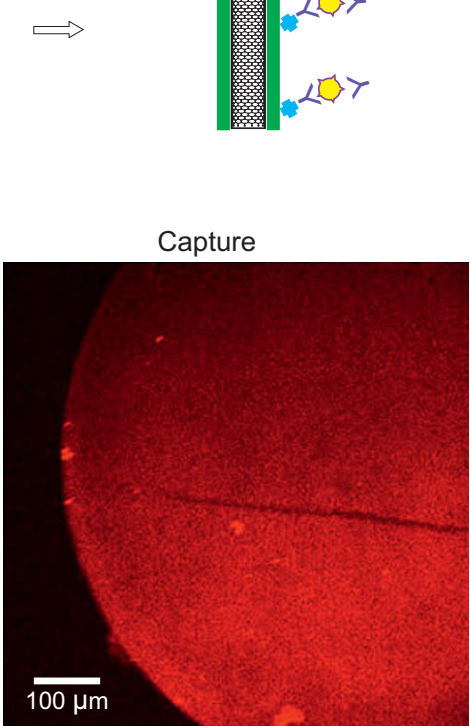

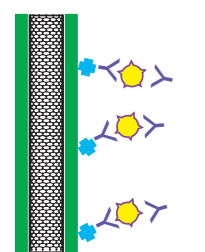

Figure 3 Bioparticle capture with a functionalized LbL-coated nanoporous element. (a) Illustration of the protocol used for PSA (100 ng $\mathrm{mL}^{-1}$ ) capture. Acid treatment after LbL assembly protonates and exposes reactive amine groups. The film is then reacted with the desired chemistries to achieve PSA capture and detection by using Qdots. (b) Confocal Z-slices in $15 \mu \mathrm{m}$ increments (starting from the bottom of the microfluidic channel) of a section of a CNT pillar element, demonstrating both LbL coating (green, FITC, $488 \mathrm{~nm}$ ) and capture (red, Qdot, 605 $\mathrm{nm}$ ). (c) Mid-plane Z-slices of control (left) and capture (right) devices representative of a multi-device series. The control experiment consists of rinsing the LbL device with blocking buffer and then proceeding directly to incubating with Qdots. 
the average intra-CNT spacing penetrate the forest and can be isolated on the forests' internal surfaces by using biomolecular recognition, and particles that are larger than the inter-element spacing are filtered. These complementary mechanisms inherent in a single element, combined with our new ability to change the intra-CNT spacing via LbL on the nanoscale, allow for the possibility of simultaneous multiscale separation across even larger bioparticle size ranges and chemistries, toward particles $\leq 80 \mathrm{~nm}$, such as viruses, proteins, and exosomes.

Modeling has suggested that changing the CNT elements' geometry to optimize the flow interception at a given pressure drop and fluid volumetric flow rate enhances polymer and bioparticle interception with the CNT surfaces ${ }^{67}$. The recorded intensity via confocal imaging is likely to be conservative and may underestimate the count because of several factors: (1) Fabrication of the microfluidic devices requires inverted imaging, i.e., through the PDMS because of the opacity of silicon. (2) The PDMS cap can vary in thickness depending on soft lithography fabrication. Thus, there is higher variability across the light intensity data because of the reflection of the excitation light from the silicon substrate and light scattering through the PDMS cap. Future work should focus on changing the CNT element geometry and the substrate used for the fabrication or configuration necessary for imaging to increase the device repeatability. One possibility for enhancement is to use quartz ${ }^{68,69}$ rather than silicon as the base substrate, which would avoid optical reflection or light scattering by the PDMS.

A key future application of the materials and technique demonstrated here is to enhance capture in microfluidic devices by enhancing interaction with 3D functionalized surfaces to improve capture and detection of nanoscale bioparticles, such as viruses $(<100 \mathrm{~nm})$, exosomes $(30-100 \mathrm{~nm})$, or rare proteins $(\sim 10 \mathrm{~nm})$. Such markers contain important information about disease state and progression. As a first step toward this goal we demonstrated polyelectrolyte multilayer coating on individual CNTs in nanoporous arrays, by using PAH-Fluor and SPS. The wide-ranging functionality of assembly materials makes it possible to tailor, at the nanoscale, the physical properties including the mechanical, electrical, and optical properties of the coatings as well as chemical functionality. Our findings provide a versatile platform for functionally tailoring and grading nanoporous scaffolds for microfluidic applications. Although the work presented here demonstrates this capability by using aligned CNT (textured) scaffolds, the technique developed may also be applied to other nanoporous material scaffolds, such as carbon nanofibers, nanowires, or aerogels, to control interfaces and surfaces in both 3D and 2D interfacial configurations.

\section{ACKNOWLEDGEMENTS}

This work was supported by the MRSEC Program of the National Science Foundation under award number DMR-0819762. The authors thank the Microsystems Technology Laboratory (MTL) at MIT for their valuable time and insight into processing techniques and instruction.

\section{CONFLICT OF INTERESTS}

The authors declare no conflict of interest.

\section{REFERENCES}

1 Guarini K, Black C, Milkove $\mathrm{K}$ et al. Nanoscale patterning using self-assembled polymers for semiconductor applications. Journal of Vaccuum Science and Technology B 2001; 19: 2784-2788.

2 Lee $\mathrm{H}$, Jung G-Y. Wafer to wafer nano-imprinting lithography with monomer based thermally curable resin. Microelectronic Engineering 2005; 77: 168-174.

3 Oh $Y$, Choi C, Hong D et al. Magnetically guided nano-micro shaping and slicing of silicon. Nano Letters 2012; 12: 2045-2050.
4 Aricò AS, Bruce $P$, Scrosati B et al. Nanostructured materials for advanced energy conversion and storage devices. Nature Materials 2005; 4: 366-377.

5 Nazar L, Goward G, Leroux F et al. Nanostructured materials for energy storage. International Journal of Inorganic Materials 2001; 3: 191-200.

6 Liu C, Li F, Ma L et al. Advanced materials for energy storage. Advanced Energy Materials 2010; 22: E28-E62.

7 Li Y, Somorjai G. Nanoscale advances in catalysis and energy applications. Nano Letters 2010; 10: 2289-2295.

8 Ozbay E. Plasmonics: Merging photonics and electronics at nanoscale dimensions. Science 2006; 311: 189-193.

9 Barone P, Baik S, Heller DA et al. Near-infrared optical sensors based on singlewalled carbon nanotubes. Nature Materials 2004; 4: 86-92.

$10 \mathrm{Ibn}$-Elhaj M, Schadt M. Optical polymer thin films with isotropic and anisotropic nano-corrugated surface topologies. Nature 2001; 410: 796-799.

11 Shipway A, Katz E, Willner I. Nanoparticle arrays on surfaces for electronic, optical, and sensor applications. Chemical Physics and Physical Chemistry 2000; 1: 18-52.

12 Caruso F. Nanoengineering of particle surfaces. Advanced Energy Materials 2001; 13: 11-22.

13 West J, Halas N. Engineered nanomaterials for biophotonoics applications: Improving ensing, imaging, and therapeutics. Annual Review of Biomedical Engineering 2003; 5: 285-292.

14 Frey N, Peng S, Cheng K et al. Magnetic nanoparticles: Synthesis, functionalization, and applications in bioimaging and magnetic energy storage. Chemical Society Reviews 2009; 38: 2532-2542.

15 Castner D, Ratner B. Biomedical surface science: Foundations to frontiers. Surface Science 2002; 500: 28-60.

16 Roach P, Farrar D, Perry C. Surface tailoring for controlled protein adsorption: Effect of topography at the nanometer scale and chemistry. Journal of American Chemical Society 2006; 128: 3939-3945.

17 Koo O, Rubinstein I, Onyuksel H. Role of nanotechnology in targeted drug delivery and imaging: A concise review. Nanomedicine: Nanotechnology, Biology and Medicine 2005; 1: 193-212.

18 Stensballe A, Andersen S, Jensen O. Characterization of phosphoproteins from electrophoretic gels by nanoscale Fe(III) affinity chromatography with off-line mass spectrometry analysis. Proteomics 2001; 1: 207-222.

19 Melechko A, Merkulov VI, McKnight TE et al. Vertically aligned carbon nanofibers and related structures: Controlled synthesis and directed assembly. Journal of Applied Physics 2005; 97: 041301.

20 Breuer O, Sundararaj U. Big returns from small fibers: A review of polymer/ carbon nanotube composites. Polymer Composites 2004; 25: 630-645.

21 Jong K, Geus J. Carbon nanofibers: Catalytic synthesis and applications. Catalysis Reviews 2007; 42: 481-510.

22 Salvetat J-P, Bonard J-M, Thomson NH et al. Mechanical properties of carbon nanotubes. Applied Physics A: Materials Science \& Processing 1999; 69: 252-260.

23 Ruoff R, Qian D, Liu W. Mechanical properties of carbon nanotubes: Theoretical predictions and experimental measurements. Comptes Rendus Physique 2003; 4: 993-1008.

24 Yakobson B, Avouris P. Mechanical properties of carbon nanotubes. Topics in Applied Physics 2001; 80: 287-327.

25 Volder M, Tawfick S, Baughman R et al. Carbon nanotubes: Present and future commercial applications. Science 2013; 339: 535-539.

26 Dresselhaus M, Dresselhaus G, Charlier J et al. Electronic, thermal and mechanical properties of carbon nanotubes. Philosophical Transactions of the Royal Society A: Mathematical, Physical and Engineering Sciences 2004; 362: 2065-2098.

27 Nihei $M$, Kawabata A, Kondo D et al. Electrical properties of carbon nanotube bundles for future via interconnects. Journal of Applied Physics 2005; 44: 1626-1628.

28 Ebbesen $\mathrm{T}$, Lezec $\mathrm{HJ}$, Hiura $\mathrm{H}$ et al. Electrical conductivity of individual carbon nanotubes. Nature 1996; 382: 54-56.

29 Kataura H, Kumazawa Y, Maniwa $\mathrm{Y}$ et al. Optical properties of single-wall carbon nanotubes. Synthetic Metals 1999; 103: 2255-2258.

30 Liu L, Ma W, Zhang Z. Macroscopic carbon nanotube assemblies: Preparation, properties, and potential applications. Small 2011; 7: 1504-1520.

31 Schnorr JM, Swager TM, Emerging applications of carbon nanotubes. Chemistry of Materials 2011; 23: 646-657.

32 Chen G, Fachin F, Colombini E et al. Nanoporous micro-element arrays for particle interception in microfluidic cell separation. Lab on a Chip 2012: 12: 3159-3167.

33 Fachin F, Chen G, Toner M, Wardle B. Integration of bulk nanoporous elements in microfluidic devices with application to biomedical diagnostics. Journal of Microelectromechanical Systems 2011; 20: 1428-1438.

34 Decher G. Fuzzy nanoassemblies: Toward layered polymeric multicomposites. Science 1997; 277: 1232-1237.

35 Michel M, Toniazzo V, Ruch D et al. Deposition mechanisms in layer-by-layer or step-by-step deposition methods: From elastic and impermeable films to soft membranes with ion exchange properties. ISRN Materials Science 2012; 2012: $1-13$. 
36 Tang Z, Wang Y, Podsiadlo $\mathrm{P}$ et al. Biomedical applications of layer-by-layer assembly: From biomimetics to tissue engineering. Advanced Materials 2006; 18 3203-3224.

37 Monge C, Almodovar J, Boudou T et al. Spatio-temporal control of LbL films for biomedical applications: From 2D to 3D. Advanced Healthcare Materials 2015; 4 811-830.

38 Richardson JJ, Björnmalm M, Caruso F. Technology-driven layer-by-layer assembly of nanofilms. Science 2015; 348: doi: 10.1126/science.aaa2491.

39 Skorb E, Volkova AV, Andreeva DV. Layer-by-layer approach for design of chemical sensors and biosensors. Current Organic Chemistry 2015; 19: 1097-1116.

40 Costa RR, Mano JF. Polyelectrolyte multilayered assemblies in biomedical technologies. Chemical Society Reviews 2014; 43: 3453-3479.

41 Ariga K, Lvov YM, Kawakami K et al. Layer-by-layer assembled shells for drug delivery. Advanced Drug Devliery Reviews 2011; 63: 762-771.

42 DeRocher J, Mao P, Han J et al. Layer-by-layer assembly of polyelectrolytes in nanofluidic devices. Macromolecules 2010; 43: 2430-2437.

$43 \mathrm{Kim}$ JY, DeRocher JP, Mao P et al. Formation of nanoparticle-containing multilayers in nanochannels via layer-by-layer assembly. Chemistry of Materials 2010; 22: 6409-6415.

44 Wang Y, Alexandra SA, Caruso F. Template synthesis of nanostructured materials via layer-by-layer assembly. Chemistry of Materials 2008; 3: 848-858.

45 Azzaroni O, Lau KH. Layer-by-layer assemblies in nanoporous templates: Nanoorganized design and applications of soft nanotechnology. Soft Matter 2011; 19: 8709-8724.

46 Komatsu T. Protein-based nanotubes for biomedical applications. Nanoscale 2012; 4: 1910-1918.

47 Sung WC, Chang CC, Makamba $\mathrm{H}$ et al. Long-term affinity modification on poly (dimethylsiloxane) substrate and its application for ELISA analysis. Analytical Chemistry 2008; 80: 1529-1535.

48 Weng $\mathrm{CH}$, Huang TB, Huang $\mathrm{CC}$ et al. A suction-type microfluidic immunosensing chip for rapid detection of the dengue virus. Biomedical Microdevices 2011; 13 585-595.

49 Kirchof K, Andar A, Yin HB et al. Polyelectrolyte multilayers generated in a microfluidic device with $\mathrm{pH}$ gradients direct adhesion and movement of cells. Lab on a Chip 2011; 11: 3326-3335.

50 Barker S, Ross D, Tarlov $M$ et al. Control of flow direction in microfluidic devices with polyelectrolyte multilayers. Analytical Chemistry 2000; 72: 5925-5929.

51 Lee J, Stein IY, Devoe ME et al. Impact of carbon nanotube length on electron transport in aligned carbon nanotube networks. Applied Physics Letters 2015 106: 053110.

52 Stein IY, Lachman N, Devoe ME et al. Exohedral physisorption of ambient moisture scales non-monotonically with fiber proximity in aligned carbon nanotube arrays. ACS Nano 2014; 8: 4591-4599.

53 Qin D, Xia Y, Whitesides G. Soft lithography for micro- and nanoscale patterning Nature Protocols 2010; 5: 491-502.

54 Vaisman L, Wagner $\mathrm{H}$, Marom $\mathrm{G}$. The role of surfactants in dispersion of carbon nanotubes. Advances in Colloid and Interface Science 2006; 37: 128-130.
55 Bystrzejewski M, Huczkob A, Langeb $\mathrm{H}$ et al. Dispersion and diameter separation of multi-wall carbon nanotubes in aqueous solutions. Journal of Colloid and Interface Science 2010; 345: 138-142.

56 Ma P-C, Siddiquia NA, Marom G et al. Dispersion and functionalization of carbon nanotubes for polymer-based nanocomposites: A review. Composites Part A: Applied Science and Manufacturing 2010; 41: 1345-1367.

57 Itano K, Choi JY, Rubner MF. Mechanism of the pH-induced discontinuous swelling/deswelling transitions of poly(allylamine hydrochloride)-containing polyelectrolyte multilayer films. Macromolecules 2005; 38: 3450-3460.

58 Lichter JA, Rubner MF. Polyelectrolyte multilayers with intrinsic antimicrobial functionality: The importance of mobile polycations. Langmuir 2009; 25: 7686-7694.

59 Polak R, Bradwell GM, Gilbert JB et al. Optimization of amine-rich multilayer thin films for the capture and quantification of prostate-specific antigen. Langmuir 2015; 31: 5479-5488.

$60 \mathrm{Kim} \mathrm{SJ}$, Han J. Self-sealed vertical polymeric nanoporous junctions for highthroughput nanofluidic applications. Analytical Chemistry 2008; 80: 3507-3511.

61 Stuart Cohen MA, Hoogendam CW, De Keizer A. Kinetics of polyelectrolyte adsorption. Journal of Physics: Condensed Matter 1997; 9: 7767.

62 Scott FH. Elements of chemical reaction engineering. 3rd ed. Prentice Hall PTR, Upper Saddle River, NJ, USA, 1999.

63 Tamayol A, Bahrami M. Transverse permeability of fibrous porous media. Physical Review E 2011; 84: 046314.

64 Ferreira M, Rubner MF. Molecular-level processing of conjugated polymers. 1. layer-by-layer manipulation of conjugated polyions. Macromolecules 1995; 28: 7107-7114.

65 Shahsavari S, McKinley GH. Interception efficiency in flow of power-law fluids past confined porous bodies. 67th Annual Meeting of the APS Division of Fluid Dynamics. 23-25 Nov 2014; San Francisco, CA, USA; 2014: H3. 00004.

66 Lilja H, Ulmert D, Vickers A. Prostate-specific antigen and prostate cancer: Prediction, detection and monitoring. Nature Reviews Cancer 2008; 8: 268-278.

67 Shahsavari S, Wardle BL, McKinley GH. Interception efficiency in two-dimensional flow past confined porous cylinders. Chemical Engineering Science 2014; 116: 752-762.

68 Zhang WD, Wen Y, Tjiu WC et al. Growth of vertically aligned carbon-nanotube array on large area of quartz plates by chemical vapor deposition. Applied Physics A: Materials Science \& Processing 2001; 74: 419-422.

69 Terrado $E$, Redrado $M$, Muñoz E et al. Aligned carbon nanotubes grown on alumina and quartz substrates by a simple thermal CVD process. Diamond and Related Materials 2006; 15: 1059-1063.

(c) (i)

This work is licensed under a Creative Commons Attribution 4.0 Unported License. The images or other third party material in this article are included in the article's Creative Commons license, unless indicated otherwise in the credit line; if the material is not included under the Creative Commons license, users will need to obtain permission from the license holder to reproduce the material. To view a copy of this license, visit http://creativecommons. org/licenses/by/4.0

Supplementary information for this article can be found on the Microsystems \& Nanoengineering website (http://www.nature.com/micronano). 